\title{
Experience of propofol sedation in a UK ERCP practice: lessons for service provision
}

\author{
D Joshi, ${ }^{1}$ B Paranandi, ${ }^{1}$ G El Sayed, ${ }^{1}$ J Down, ${ }^{2}$ G J Johnson, ${ }^{1}$ \\ M H Chapman, ${ }^{1}$ S P Pereira, ${ }^{1}$ G J M Webster ${ }^{1}$
}

\author{
${ }^{1}$ Department of \\ Gastroenterology, University \\ College London Hospitals, \\ London, UK \\ ${ }^{2}$ Department of Anaesthesia, \\ University College London \\ Hospitals, London, UK
}

\section{Correspondence to} Dr D Joshi, Department of Gastroenterology, University College Hospital, 250 Euston Road, London NW1 2PG, UK d.joshi@nhs.net

Received 30 June 2014 Revised 11 August 2014 Accepted 18 August 2014 Published Online First 10 September 2014

\section{CrossMark}

To cite: Joshi $D$, Paranandi $B$, El Sayed $G$, et al. Frontline Gastroenterology 2015;6: 32-37.

\begin{abstract}
Objective Endoscopic retrograde cholangiopancreatography (ERCP) in the UK has been historically performed under conscious sedation. However, given the increasing complexity of cases, the role of propofol-assisted ERCP (propERCP) is increasing. We describe our experience of propERCP and highlight the importance of this service.
\end{abstract}

Design Our prospective ERCP database was interrogated between January 2013 and January 2014. Data collection included procedural information, patient demographics, American Association of Anaesthesiologists (ASA) status, Cotton grade of endoscopic difficulty and endoscopic and anaesthetic complications. Comparison was made with patients undergoing conscious sedation ERCP (sedERCP).

Results 744 ERCPs were performed in 629 patients (53\% male). 161 ERCPs were performed under propofol. PropERCP patients were younger compared with the sedERCP group (54 vs 66 years, $p<0.0001)$ but ASA grade $1-2$ status was similar ( $84 \%$ vs $78 \%, p=0.6)$. An increased number of Cotton grade 3-4 ERCPs were performed in the propERCP group (64\% vs $34 \%, \mathrm{p}<0.0001)$. Indications for propERCP included sphincter of Oddi manometry (27\%), previously poorly tolerated sedERCP (26\%), cholangioscopy (21\%) and patient request (8\%). $77 \%$ of cases were elective, $12 \%$ were urgent day-case transfers and $11 \%$ were urgent inpatients. 59\% of cases were tertiary referrals. ERCP was completed successfully in $95 \%$ of cases. Anaesthetic and endoscopic complications were comparable between the two groups (5\% and $7 \%$ vs $3 \%$ and $5 \%$ ). Where sedERCP had been unsuccessful due to patient intolerance, the procedure was completed successfully using propofol.

Conclusions PropERCP is safe and is associated with high endoscopic success. The need for
propERCP is likely to increase given patient preference and the high proportion of complex procedures being undertaken. All endoscopy units should look to incorporate propofolassisted endoscopy into aspects of their services.

\section{INTRODUCTION}

Endoscopic retrograde cholangiopancreatography (ERCP) is an important diagnostic and therapeutic tool in the management of biliary and pancreatic disorders. In the majority of units within the UK, ERCP is performed under conscious sedation using an intravenous combination of midazolam and an opioid (usually fentanyl or pethidine) delivered by the endoscopist. ${ }^{1}$

Conscious sedation has been reported to be inadequate for up to $14 \%$ of patients undergoing ERCP, ${ }^{2}$ and a poorly tolerated procedure can lead to ERCP failure and possibly procedure-associated complications. The 2004 British Society of Gastroenterology (BSG) audit of ERCP in the UK highlighted that 33\% (1484 of 4521 ) of patients undergoing conscious sedation with midazolam received a dose of $>5.5 \mathrm{mg}$, with approximately $8 \%$ of patients requiring the administration of reversal agents (flumazenil or naloxone). ${ }^{3}$ A previous audit by our unit demonstrated that intolerance of conscious sedation was a significant factor in ERCP failure. ${ }^{4}$ In 2011, the BSG issued guidance in conjunction with the Royal College of Anaesthetists (RCoA) regarding the use of propofol sedation without the need for tracheal intubation in patients undergoing ERCP and other complex endoscopic procedures. ${ }^{5}$ These guidelines highlighted the minimum requirements for all endoscopic units wanting to deliver this service. 
Attractions of propofol (2,6-di-isopropofol) in endoscopic procedures include its rapid onset of action and short duration of effect. ${ }^{6}$ In the UK, propofol sedation is delivered by an anaesthetist with appropriate anaesthetic support.

The endoscopy department at University College London Hospitals (UCLH) is a secondary and tertiary referral centre for pancreatico-biliary endoscopy, with specific complex procedures including single-operator cholangioscopy, sphincter of Oddi manometry (SOM), pancreatic endotherapy and therapeutic endoscopic ultrasound. Since January 2011, we have had the availability of two propofol-assisted ERCP (propERCP) lists per week in order to provide these services.

Here, we describe our experience of propERCP. Our aims were to describe patient demographics, indications for propERCP, complications both endoscopic and anaesthetic, and highlight the importance of this service for units offering ERCP, including the financial implications.

\section{METHODS}

\section{Study population}

All patients (adults $>18$ years) undergoing ERCP at UCLH between January 2013 and December 2013 were included. Patients were identified using a prospectively maintained ERCP database. All ERCP procedures were consultant delivered or in-room supervised, using a TJFV or JFV video duodenoscope (Olympus; Olympus Keymed, Southend-on-sea, Essex, UK). Data collection included patient demographics, indication for ERCP, American Society of Anaesthesiologists (ASA) status (I-IV), Cotton grade of endoscopic difficulty $(1-4)^{7}$ (table 1), procedural information and endoscopic and anaesthetic complications. As prophylaxis against ERCP-related pancreatitis all patients received rectal indomethacin, unless contraindicated.

\section{Propofol-assisted ERCP}

Two propERCP lists each week were delivered within the endoscopy department during the study period. Sedation was delivered by a dedicated group of consultant anaesthetists all experienced in propofol sedation for endoscopic procedures, with the support of an operating department assistant or anaesthetic nurse. All patients received electrocardiographic, pulse oximetry and non-invasive blood pressure monitoring during the procedure. An OmniLine Guardian mouth guard was used and end-tidal $\mathrm{CO}_{2}$ was monitored via the mouth guard in order to comply with the RCoA guidelines for anaesthesia. Patients were positioned in the semiprone position. The target was to produce deep sedation defined as loss of verbal contact but purposeful response to repeated or painful stimuli. Propofol $1 \%$ was administered in combination with remifentanyl $(2 \mathrm{mcg} / \mathrm{mL})$ in all propERCP patients. It was delivered by target controlled intravenous infusion (MARSH model). An initial effect site
Table 1 Cotton grades of difficulty of endoscopic retrograde cholangiopancreatography (ERCP) procedures ${ }^{7}$

\begin{tabular}{ll}
\hline Grade & Procedure \\
\hline 1 & Deep cannulation of duct of interest, main papilla or sampling \\
& Biliary stent removal or exchange \\
& Biliary stone extraction $<10 \mathrm{~mm}$ \\
& Treatment of biliary leaks \\
& Treatment of extrahepatic strictures (benign or malignant) \\
& Placement of prophylactic pancreatic stents \\
& Biliary stone extraction $>10 \mathrm{~mm}$ \\
& Minor papilla cannulation in divisum and therapy \\
& Removal of internally migrated biliary stents \\
& Intraductal imaging, biopsy or fine-needle aspiration \\
& Management of acute or recurrent pancreatitis \\
& Treatment of pancreatic strictures \\
& Removal of pancreatic stones that are mobile and $<5$ mm \\
& Treatment of hilar tumours \\
& Treatment of benign biliary strictures, hilum and above \\
& Management of sphincter of Oddi dysfunction (with or without \\
& mannometry) \\
& Removal of internally migrated pancreatic stents \\
& Intraductal image-guided therapy (eg, photodynamic therapy) \\
& Removal of intrahepatic stones \\
& Pseudocyst drainage or necrosectomy \\
& Ampullectomy \\
ERCP after a Whipple procedure or Roux-en-Y bariatric surgery
\end{tabular}

One grade should be added (for a maximum grade of 4 ) for procedures performed after normal working hours, children under 3 years of age, in post-Billroth II gastrectomy patients, or for procedures that have previously failed.

concentration was set at $2 \mathrm{mcg} / \mathrm{mL}$. The infusion was then titrated against patient effect. A small dose of midazolam (1-2 mg) was delivered in some patients prior to the infusion at the discretion of the anaesthetist. Patients who required de novo intubation and ventilation were excluded as were procedures performed either in day surgery or theatres.

All patients who received propofol had undergone a preassessment review prior to the procedure. This included routine blood tests, an ECG and review of the patient's medical and drug history. Most preassessments involved a face-to-face consultation, but there was the facility of telephone assessment if anaesthetic needs/comorbidities were minimal. Most patients had a planned overnight admission postprocedure given the long distances travelled.

\section{Statistical analysis}

Continuous variables were expressed as median and range (minimum and maximum) and compared by non-parametric tests. Categorical variables were compared using the $\chi^{2}$ or Fisher's exact test. Comparison was made between the sedation ERCP (sedERCP) and the propERCP groups. A two-tail $\mathrm{p}$ value $<0.05$ was considered to indicate statistical significance. Analysis was performed using SPSS for Windows V.20 (SPSS, Chicago, Illinois, USA).

\section{RESULTS}

During the study period, a total of 744 ERCPs were performed in 629 patients (53\% male). Median age of 
patients was 63 years (18-96). Nearly half of the patients (49\%) were undergoing their index ERCP. Median ASA status was 2 (1-4) and median Cotton grade of endoscopic difficulty was $2(1-4) .556$ were performed using conscious sedation. Propofol sedation was used in 161 ERCPs, while a further 27 ERCPs required a general anaesthetic (GA) with intubation and ventilation (median ASA grade 3, range 14); 70\% $(n=19)$ were ASA grade 3-4. Indications for full GA included high body mass index $(B M I)(n=13)$ and cardio/respiratory disease $(n=14)$.

\section{Propofol-assisted ERCP}

Patients in the propERCP group were younger compared with the sedERCP group (median 54 years, range $18-88$ vs median 66 years, range 20-96, $\mathrm{p}<0.0001$ ), but ASA grade $1-2$ status was similar between the two groups ( $84 \%$ vs $78 \%, \mathrm{p}=0.6)$ (table 2). The propERCP group were less likely to have a virgin papilla ( $37 \%$ vs $53 \%, \mathrm{p}=0.02)$. An increased number of Cotton grade 3-4 ERCPs were performed in the propERCP group (64\% vs $34 \%$, $\mathrm{p}<0.0001$ ).

Indications for propERCP are listed in table 3 and included SOM ( $\mathrm{n}=43,27 \%)$; previously poorly tolerated ERCP under conscious sedation $(\mathrm{n}=41,26 \%)$; single-operator (Spyglass) cholangioscopy (with or without electrohydraulic lithotripsy for stone therapy) $(n=33,21 \%)$ and patient request for index ERCP $(\mathrm{n}=13,8 \%)$. Other indications $(\mathrm{n}=5,3 \%)$ included previous surgery and a need for combined radiological/endoscopic procedure.

Elective cases accounted for $77 \%$ of propERCP, $12 \%$ were urgent day-case transfers from referring hospitals and $11 \%$ were urgent inpatients. Tertiary referrals accounted for $59 \%$ of the cases, $11 \%$ of which had previously unsuccessful ERCPs. In the 18 patients who had had a previously failed ERCP procedure, eight of the cases were due to failed cannulation, seven due to patient intolerance and three due to a drop in patient oxygen saturations. Where a previous ERCP had failed due to patient intolerance under

Table 2 Comparison between the conscious sedation ERCP and propofol-assisted ERCP group

\begin{tabular}{lccc}
\hline & sedERCP & propERCP & p Value \\
\hline Age (years)* & $66(20-96)$ & $54(18-88)$ & $<0.0001$ \\
Male (n) $(\%) \dagger$ & $266(48 \%)$ & $83(52 \%)$ & 0.7 \\
Virgin papillae $(n)(\%) \dagger$ & $294(53 \%)$ & $60(37 \%)$ & 0.02 \\
ASA status 1-2 (n) $(\%) \dagger$ & $433(78 \%)$ & $135(84 \%)$ & 0.6 \\
Cotton grade 3-4 (n) $(\%) \dagger$ & $189(34 \%)$ & $103(64 \%)$ & $<0.0001$ \\
\hline
\end{tabular}

Values expressed as median with range (min-max) or frequency and \%. *Mann-Whitney.

$+\chi^{2}$.

ASA, American Association of Anaesthesiologists; ERCP, endoscopic retrograde cholangiopancreatography; sedERCP, sedation ERCP; propERCP propofol-assisted ERCP.
Table 3 Indications for propofol-assisted endoscopic retrograde cholangiopancreatography

\begin{tabular}{ll}
\hline & $(\mathrm{n} / \%)$ \\
\hline Sphincter of Oddi manometry & $43(27 \%)$ \\
Previously intolerant of conscious sedation & $41(26 \%)$ \\
Cholangioscopy & $33(21 \%)$ \\
Previously failed selective duct cannulation & $18(11 \%)$ \\
Patient request & $13(8 \%)$ \\
Pancreatic endotherapy & $8(5 \%)$ \\
Other & $5(3 \%)$ \\
\hline
\end{tabular}

conscious sedation, the procedure was completed in all cases using propERCP. Overall, selective duct cannulation and therapy (if indicated) were completed in $95 \%$ of cases.

\section{Complications}

Anaesthetic

Anaesthetic complications or complications relating to sedation were similar between the two groups $(5 \%$ propERCP vs $3 \%$ sedERCP, $\mathrm{p}=0.3$ ). Two cardiorespiratory arrests occurred both in the sedERCP group; one patient suffered a ventricular fibrillation (VF) arrest due to severe undiagnosed and asymptomatic ischaemic heart disease and the other patient suffered a respiratory arrest following aspiration. Both patients made a full recovery.

In the propERCP group, anaesthetic complications $(n=8)$ were all related to recognised side effects of deep sedation. Two patients required a combination of airway manipulations, which included a chin lift manoeuvre, the use of a modified mask airway and the use of a nasal airway. Three patients developed transient hypotension. Three patients required bag and mask ventilation followed by endotracheal intubation and ventilation. No patient required admission to the intensive care unit for ongoing ventilation or for the need of vasopressors. The development of an anaesthetic complication in the propERCP group did not impact on the length of stay post-ERCP (median length of stay 1 day, $0-3$ days) compared with patients that did not develop an anaesthetic complication (median length of stay 1 day, $0-40$ days, $\mathrm{p}=0.6$ ).

\section{Endoscopic}

The rate of endoscopic complications was similar between the two groups ( $7 \%$ propERCP vs $5 \%$ sedERCP, $p=0.2)$. In the propERCP group, endoscopic complications included pancreatitis $(n=7,4 \%)$, perforation $(n=2,1 \%)$ and bleeding $(n=3,2 \%)$. The two cases of perforation occurred following pancreatic sphincterotomy in patients with pancreatic sphincter hypertension/fibrosis. Bleeding occurred in patients following biliary sphincterotomy all of which settled with conservative/endoscopic measures. Unplanned hospital stay occurred in 27 patients (17\%) largely 
due to ongoing abdominal pain. No deaths were observed in either group in the subsequent 30 days post-ERCP.

\section{DISCUSSION}

ERCP remains an important diagnostic and therapeutic tool in the investigation and management of hepato-pancreatico-biliary disorders. The move away from diagnostic ERCP and introduction of new technologies have led to longer and more complex procedures being undertaken.

Our study identified 161 ERCPs performed using propofol sedation, representing 22\% of our annual ERCP caseload. As expected, ERCPs performed under propofol were more complex, as demonstrated by the increased number of Cotton grade 3-4 cases, reflecting the selection of patients with expected longer and less well-tolerated procedures (eg, cholangioscopy, SOM, ERCP in altered anatomy, pancreatic endotherapy). Patients undergoing propofol sedation were younger. This may be explained by the large number of patients undergoing SOM, who anecdotally may tolerate procedure under sedation poorly. The number of ASA grade 1-2 status patients was similar between the two groups and was an expected finding because patients are not primarily selected for propofol on the basis of comorbidity. At UCLH, patients with severe comorbidity, severe respiratory disease or high BMI are more likely to have a full GA. Direct patient request accounted for $8 \%$ of cases performed using propofol. There is now an increasing awareness among patients that propofol is used and available for endoscopic procedures. Patient request for propofol sedation will continue to increase.

We used a combination of propofol and remifentanyl because of their combined ability to provide deep sedation, reflex obtundation and good operator conditions, while both being very short acting so quickly reversed on infusion cessation. These properties are important because the patients are positioned semiprone and have no airway support. Apnoea and airway obstruction are the most important risks of deep propofol sedation. In experienced hands, they are usually averted by simple airway manoeuvres (chin lift and jaw thrust) and reduction or cessation of the sedative infusion. However, in a small group (eight patients in our study), further airway adjuncts and ventilatory support were required up to and including intubation and ventilation. For this reason, it is our policy to have all the equipment in the room, checked and ready for use, to deliver general anaesthesia. The narrow therapeutic window and serious nature of the complications of deep propofol sedation warrant the use of senior anaesthetists, experienced with its use and accompanied by trained assistants, in our opinion. Patients at high risk of airway complications (the morbidly obese and those with known or suspected difficult airways) and those with severe cardiorespiratory compromise should be considered for formal general anaesthesia with endotracheal intubation due to the increased level airway and ventilatory control it offers.

Midazolam, in low dose, was used at the discretion of the anaesthetist for two indications. First, it was used as an anxiolytic and second, to reduce the incidence of awareness. The incidence of awareness during propofol sedation is low and no patient in our study complained of being aware during the procedure. However, as it is not general anaesthesia, no guarantee regarding awareness should be given to patients prior to propofol sedation.

In a study of nearly 800 patients undergoing endoscopic procedures with use of propofol, 336 of whom underwent ERCP, $87 \%$ of patients showed no response to endoscopic intubation. ${ }^{8}$ Premature termination of the procedure occurred in $<1 \%$ of cases. Although this study provided no specific data regarding (propERCP), the authors concluded that propofol can be used safely for endoscopic procedures when administered by a trained professional. The study did highlight the need for caution and an increased risk of airway modifications in male patients, patients' ASA $\geq 3$ and patients with an increased BMI. ${ }^{8}$ UK guidelines mandate that the delivery of propofol during endoscopic procedures is by an experienced anaesthetist. $^{5}$

Nearly $60 \%$ of our cases were tertiary referrals from other hospitals. The majority of these referrals were for complex procedures not offered in the referring hospitals. However, $11 \%$ of cases had also failed previously at the referring hospital. The routine availability of a propERCP lists is, therefore, an important facility if one is offering a service. The great majority of propofol ERCPs were non-urgent, and the availability of propERCP may not be essential for all ERCP units, as long as this service is available locally/ within a network. This is important when developing local pathways and local networks for ERCP provision.

In France and Germany, propofol sedation is the standard of care for ERCP. Some centres within the UK have now adopted propERCP as their standard of care. ${ }^{9}$ The use of propofol reduces patient movement and discomfort, which possibly may then reduce the risk of procedure-related complications, and be associated with higher postprocedure patient satisfaction compared with conscious sedation. ${ }^{10}$ In our data, endoscopic complication rates were comparable between the two groups (7\% propERCP vs $5 \%$ sedERCP, $p=0.2$ ). The patient cohort in this study falls into a high-risk category for endoscopic complications and consists of technically challenging cases as represented by the large numbers of Cotton grade 3-4 cases across both groups.

Although our unplanned hospital length of stay (ie, greater than the anticipated overnight stay) in the 
propERCP group may seem high, all 27 patients that were identified had undergone SOM. SOM is now a declining indication in our unit following the publication of the EPISOD study. ${ }^{11}$ Results from a Cochrane review have demonstrated a better recovery profile among patients receiving propofol, but no difference with regards to safety, when compared with the use of midazolam and opioid. ${ }^{12}$ Our data demonstrated similar complication rates in both groups. Anaesthetic complications occurred in eight patients, all of which related to recognised complications of deep sedation. Although three patients required intubation and ventilation, no patient required admission to the intensive care unit for ongoing respiratory support.

At our institution, we have seven dedicated ERCP lists, two of which are propofol assisted. The remaining five lists are therefore performed with the use of conscious sedation only. The cost of a consultant anaesthetist and a band 6 operating department assistant (ODA) for $4 \mathrm{~h}$ at UCLH is approximately $£ 1000.00$ per list. As all cases are performed within the endoscopy department, no extra costs are incurred. This, however, will be an underestimate as it does not take into account the cost of the preassessment clinic or the reduced numbers of procedures per list. All of these aspects need to be considered and incorporated when calculating the financial implications of providing this service.

\section{Significance of this study}

\section{What is already known?}

- More complex procedures are being undertaken at endoscopic retrograde cholangiopancreatography (ERCP).

- ERCP under conscious sedation can lead to complications and procedure failure.

What are the new findings?

- Propofol assisted ERCP is safe both anaesthetically and endoscopically.

- Propofol assisted ERCP should be considered when attempting complex procedures (Cotton grade 3-4).

- The demand for propofol assisted ERCP is likely to increase.

How might it impact on clinical practice in the foreseeable future?

- The demand for propofol assisted ERCP is likely to increase as both clinicians and patients request propofol sedation.

- Endoscopy units need to have the availability of propofol assisted ERCP either in house or as part of an ERCP network.

- A close working relationship with the anaesthetic department is required to help delivering the service.
Another key finding from our study was that $8 \%$ of our cases undergoing propERCP were due to direct patient request at the index ERCP. If weekly propERCP lists are not feasible, then a monthly list may be more realistic. This strategy has been successfully introduced by units in the UK incorporating other endoscopic procedures. ${ }^{13}$

In conclusion, propERCP may enhance patient outcome within an ERCP service and may be particularly useful if one is undertaking complex ERCPs (Cotton grade $\geq 3$ ). PropERCP appears safe with comparable endoscopic and anaesthetic/sedation-related complication rates. The demand for propERCP is likely to rise as patients and clinicians request propofol sedation for ERCP. The provision and the availability of propERCP need to be addressed by units and networks providing this service.

Contributors DJ and GJM wrote, edited and revised the article. $\mathrm{BP}$ and GES helped with the collection of data. JD, GJJ, MHC and SPP helped edit and revise the article.

Competing interests None.

Provenance and peer review Not commissioned; externally peer reviewed.

\section{REFERENCES}

1 Goulson DT, Fragneto RY. Anesthesia for gastrointestinal endoscopic procedures. Anesthesiol Clin 2009;27:71-85.

2 Raymondos K, Panning B, Bachem I, et al. Evaluation of endoscopic retrograde cholangiopancreatography under conscious sedation and general anesthesia. Endoscopy 2002;34:721-6.

3 Williams EJ, Taylor S, Fairclough P, et al. Are we meeting the standards set for endoscopy? Results of a large-scale prospective survey of endoscopic retrograde cholangio-pancreatograph practice. Gut 2007;56:821-9.

4 Church NI, Seward E, Pereira SP, et al. Success of repeat ERCP following initial therapeutic failure. Gut 2007;56(Suppl 2): a1-a163.

5 Guidance for the use of propofol sedation for adult patients undergoing endoscopic retrograde cholangiopancreatography (ERCP) and other complex upper GI endoscopic procedures. On behalf of the Joint Royal College of Anaesthetists (RCoA) and British Society of Gastroenterology (BSG) Working Party. April 2011.

6 Faulx AL, Vela S, Das A, et al. The changing landscape of practice patterns regarding unsedated endoscopy and propofol use: a national Web survey. Gastrointest Endosc 2005;62:9-15.

7 Cotton PB, Eisen G, Romagnuolo J, et al. Grading the complexity of endoscopic procedures: results of an ASGE working party. Gastrointest Endosc 2011;73:868-74.

8 Cote GA, Hovis RM, Ansstas MA, et al. Incidence of sedation-related complications with propofol use during advanced endoscopic procedures. Clin Gastroenterol Hepatol 2010;8:137-42.

9 Lordan JT, Woods J, Keeling P, et al. A retrospective analysis of benzodiazepine sedation vs. propofol anaesthesia in 252 patients undergoing endoscopic retrograde cholangiopancreatography. HPB (Oxford) 2011;13:174-7. 
10 Jung M, Hofmann C, Kiesslich R, et al. Improved sedation in diagnostic and therapeutic ERCP: propofol is an alternative to midazolam. Endoscopy 2000;32:233-8.

11 Cotton PB, Durkalski V, Romagnuolo J, et al. Effect of endoscopic sphincterotomy for suspected sphincter of Oddi dysfunction on pain-related disability following cholecystectomy: the EPISOD randomized clinical trial. JAMA 2014;311:2101-9.
12 Garewal D, Powell S, Milan SJ, et al. Sedative techniques for endoscopic retrograde cholangiopancreatography. Cochrane Database Syst Rev 2012;6:CD007274.

13 Joshi D, Austin M, Bate T, et al. An increasing demand for enhanced sedation endoscopy: a single centre experience. Gut 2014;63(Suppl 1):PTH-031, A222. 